Check for updates

Cite this: RSC Adv., 2019, 9, 13586

Received 18th February 2019 Accepted 10th April 2019

DOI: 10.1039/c9ra01251f rsc.li/rsc-advances

\section{Prebiotic effects of alfalfa (Medicago sativa) fiber on cecal bacterial composition, short-chain fatty acids, and diarrhea incidence in weaning piglets}

\author{
Seidu Adams, (D) ${ }^{\text {ab }}$ Kong Xiangjie, ${ }^{\text {ab }}$ Jiang Hailong, ${ }^{\text {abc }}$ Qin Guixin, ${ }^{\text {abc }}$ \\ Fredrick Leo Sossah iD d and Che Dongsheng*abc
}

Dietary alfalfa fiber (AF) is conceived to modulate gut microbial richness and diversity to improve the health and growth of weaning piglets. The objective of this study was to evaluate the prebiotic effects of AF on diarrhea incidence, the production of short-chain fatty acids (SCFAs), and microbiota composition in weaning piglets. This study utilized 100 crossbred piglets (Duroc $\times$ Landrace $\times$ Yorkshire) with a body weight of $8.42 \pm 1.88 \mathrm{~kg}$ randomly assigned to the following treatments: $0.00 \% \mathrm{AF}$ meal (A), $6.00 \%$ of $\mathrm{AF}$ meal (B), $12.00 \% \mathrm{AF}$ meal (C), and $18.00 \%$ AF meal (D). The cecum samples were used to determine microbial community composition and diversity through high-throughput $16 \mathrm{~S}$ rDNA sequencing. The results of this study show that the lowest average daily gain (ADG) was observed in treatment $D$, and the highest ADG was recorded in treatment C. However there was no significant difference between the treatment groups and the control. The average daily feed intake (ADFI) was significantly higher in treatment $C$ compared to the other treatments. The feed conversion ratio was high in the control group compared to the AF treated groups. The highest diarrhea incidence was observed in treatment $A$ and the lowest diarrhea incidence was observed in treatment $C$ and $D$. The highest acetate and propionate levels were observed in treatment $B$, but there was no significant difference between the treatment groups and the control. The supplementation of AF significantly increased the butyrate level in treatment $D$ compared with treatments $A$ and $B$ but was not significantly different from treatment $C$. The Observed_species richness and Simpson diversity values of the cecum bacterial composition in the AF fed piglets were higher than the control. In addition, the Chao 1 richness and Shannon diversity increased with an increase in AF supplementation, reaching a plateau at treatment $B$ and C, then decreasing at treatment D. The Bacteroidetes, Firmicutes, Tenericutes, Proteobacteria, Cyanobacteria, Spirochaetae, Actinobacteria, Fibrobacteres, Saccharibacteria, Synergistetes, Chlamydiae, Elusimicrobia, Deferribacteres, Fusobacteria, and others were relatively abundant in all treatments. The Bacteroidetes and Firmicutes were the dominant phyla, accounting for $98 \%$ of all reads. AF treatment decreased the Bacteroidetes phylum and increased the Firmicutes phylum compared with treatment A. Therefore, the dietary inclusion of AF may decrease diarrhea incidence, increase cecal bacterial composition and richness, and consequently improve the growth performance of weaning piglets.

\section{Introduction}

The gut microbiota contains over $10^{14}$ microbial cells that collectively comprise at least 150 times more genes than their host. ${ }^{1,2}$ The composition of the gut microbiota in humans and other monogastric animals varies based on age, diet, genetic characteristics, and sex. ${ }^{1}$ Gut microbiota have some important functions in host

\footnotetext{
${ }^{a}$ College of Animal Science and Technology, Jilin Agricultural University, Changchun, 130118, China.E-mail: hljiang@jlau.edu.cn; chedongsheng@jlau.edu.cn

${ }^{b}$ Jilin Provincial Key Lab of Animal Nutrition and Feed Science, Jilin Agricultural University, Changchun, 130118, China

'Key Lab of Animal Production, Product Quality and Security, Ministry of Education, Jilin Agricultural University, Changchun, 130118, China

${ }^{d}$ College of Agronomy and Molecular Biology, China
}

health, nutrient metabolism and absorption, development of the host immune system, differentiation of the gut epithelium, and maintenance of the gut mucosal barrier functions. ${ }^{3,4}$ Previous evidence indicated that gut microorganisms can directly influence physiological conditions in animals such as improving the roles of the intestinal barrier, stimulating defence mechanisms against pathogens, improving the host immune system, increasing defensive mechanisms against inflammatory bowel diseases (IBD), ${ }^{5}$ regulating autoimmunity, producing biological metabolites, preventing obesity and regulating diabetes, and destroying cancer cells. ${ }^{\mathbf{1 , 6}}$ The interactions between the host gut-microbiota are dynamic and highly vulnerable to several environmental conditions, particularly diet. ${ }^{1}$

Alfalfa is a high-yielding perennial legume that is cultivated worldwide with rich nutritional characteristics and bioactive 
compounds. ${ }^{7,8}$ Alfalfa fiber is mainly composed of insoluble dietary fiber, such as cellulose, lignin, and xylans, representing more than $90 \%$ of the total dietary fiber composition of alfalfa. ${ }^{9,10}$ Interestingly, alfalfa meal contains $17.5 \% \mathrm{CP}, 24.1 \%$ $\mathrm{CF}$, and $1200 \mathrm{kcal} \mathrm{kg}^{-1}$ metabolizable energy. ${ }^{11}$ Recent studies reported that pigs grown on alfalfa pastures produce goodquality natural pork, which is currently gaining more attention in Europe and North America. ${ }^{12,13}$ Interestingly, for the production of good quality pork, alfalfa is used as fresh fodder in some traditional style pig production systems in China ${ }^{\mathbf{1 3}}$ and other parts of the world. AF is a high-quality protein feed; therefore, increasing its supplementation may improve the nutritional requirement of pigs and increase the growth of piglets. Few studies reported that the supplementation of natural compounds such as flavonoids extracted from alfalfa had no estrogenic impacts, which may increase the production performance, enhance antioxidants, induce anti-stress function, and eradicate free radicals from animals. ${ }^{12,14}$ Dietary fiber influence the role of the gut microbiota, as indicated by the low digestibility and availability of nutrients in feeds with high fiber levels. ${ }^{\mathbf{1 , 1 5}}$ The type of dietary fiber, soluble or insoluble, influences its effects on gut microbial composition and function. ${ }^{\mathbf{1 , 1 6 , 1 7}}$ The intake of high dietary fiber has been reported to influence energy metabolism, nutrient utilization, and subsequently results in a reduction in the performance of monogastric animals, especially pigs. ${ }^{18-20}$ Recent findings indicated that dietary fiber can prevent intestinal diseases such as constipation, diarrhea, bowel diseases, and improve intestinal health in both humans and animal subjects. ${ }^{\mathbf{1 0 2 1 , 2 2}}$ Different dietary fiber has beneficial roles in gut microbial composition and function. Such beneficial roles include SCFAs synthesis, decreased intestinal $\mathrm{pH}$, and fecal bulkiness., ${ }^{\mathbf{1 , 2 1 , 2 3}}$ It was reported that dietary fiber may selectively modulate intestinal microbiota via the stimulation of constructive microbial species and the suppression of enteric bacterial species. ${ }^{\mathbf{1 0 2 4}}$ Dietary fiber functions as a nutrient for intestinal microbiota and consequently motivates the growth of valuable intestinal bacteria, like Bifidobacterium, which are involved in the reduction in cardiovascular disorders. ${ }^{25}$

Weaning piglets are associated with a reduction in feed consumption and low nutrient metabolism due to the immature digestive systems, stress as a result of the separation from the sows and litter mates, and the sudden change from the easily digestible sow's milk to less digestible solid feed., ${ }^{12,16,17,26}$ The treatment of high-fiber diets to piglets results in an overall increase in the total empty-weight of the gut ${ }^{27}$ and an increase in gut secretions. ${ }^{28}$ Dietary fiber increased the growth performance, nutrient digestibility, and immunity in piglets, ${ }^{29,30}$ resulting in a decrease in diarrhea incidence. ${ }^{31,32}$ There was a report that dietary fiber supplementation may improve growth performance and decrease the intestinal permeability in piglets, ${ }^{31,33}$ indicated that dietary fiber extracted from Astragalus membranaceus increased growth performance, nutrient digestibility, VFA production, and immunity in piglets. However, the effects of $\mathrm{AF}$ on gut microbiota composition, health, and energy metabolism in piglets are limited.

Therefore, the objective of this study was to evaluate the prebiotic effects of AF on diarrhea incidence, SCFA production, and microbiota composition in weaning piglets. This objective was investigated by feeding different concentrations of AF to 28 day-old piglets for 48 days and the cecal samples were extracted for microbial analysis. The $16 \mathrm{~S}$ rDNA pyrosequencing technique was used to examine the prebiotic effects of AF on the cecal microbial composition, and the growth parameters of the piglets were examined.

\section{Materials and methods}

\subsection{Animal care and ethics}

All animal procedures were carried out in accordance with the Guidelines for the Care and Use of Laboratory Animals of Jilin Agricultural University, and experiments were approved by the Animal Ethics Committee of Jilin Agricultural University.

\subsection{Experimental animals, diet, and sampling}

A total of 100 crossbred piglets (Duroc $\times$ Landrace $\times$ Yorkshire) weaned at 28 days of age with a mean body weight of $8.42 \pm 1.88$ $\mathrm{kg}$ were randomly allocated to 4 treatment groups with 5 replicates per treatment and 5 pigs per replicate using the randomized complete block design. The piglets were housed in pens $(1.8 \times 1.2 \mathrm{~m})$ with a concrete slatted floor and ad libitum access to feed and water. The room temperature was maintained at $26^{\circ} \mathrm{C}$ at the time of weaning and progressively reduced to $22{ }^{\circ} \mathrm{C}$ within the first week of post weaning. The humidity was kept constant at $65-75 \%$. The piglets in each group received one of the 4 experimental diets (Table 1) with cracked corn and soybean meal as the main source of energy and protein, respectively. The diets were formulated as follows: ${ }^{34}$ the control diet of $0.00 \% \mathrm{AF}(\mathrm{A}), 6.00 \% \mathrm{AF} \operatorname{diet}(\mathrm{B}), 12.00 \% \mathrm{AF} \operatorname{diet}(\mathrm{C})$, and $18.00 \%$ AF diet (D). All nutritional components in Table 1 were supplement based on the national nutrient requirements of piglets with the exceptions of AF supplementation. Therefore, the obtained results were analysed based on the added AF levels. The experiment lasted for 48 days and 7 days of prefeeding tests. There was no antibiotic supplementation before and during the experimental trial. Piglets were weighed individually at the beginning and the end of the experiment and daily feed intake per pen was recorded. At the end of the feeding trial, 10 pigs were randomly selected from each group. After fasting overnight, the pigs were slaughtered, and the gastrointestinal tract was immediately removed. The cecal content was collected into $5 \mathrm{~mL}$ cryotubes and snap-frozen immediately in liquid nitrogen and stored at $-80{ }^{\circ} \mathrm{C}$ until further microbial and metagenomics analysis. In addition, $50 \mathrm{~mL}$ centrifuge tube of cecum digest samples were collected and frozen in $-20{ }^{\circ} \mathrm{C}$ for SCFAs analysis.

\subsection{Growth performance, diarrhea incidence, and SCFAs}

The initial body weight of the piglets was subtracted from the final body weight and divided by the days of the experiment to obtain the ADG. The ADFI was calculated by estimating the total feed intake per day. The FCR was calculated by dividing the feed intake by the body weight gain. All piglets were checked for the consistency in fecal samples as an indicator of their health 
Table 1 Shows the composition and nutritional levels of the basal diets $(\%)^{a}$

\begin{tabular}{|c|c|c|c|c|}
\hline & A & B & $\mathrm{C}$ & $\mathrm{D}$ \\
\hline \multicolumn{5}{|l|}{ Items } \\
\hline Corn & 53.50 & 50.29 & 45.00 & 40.00 \\
\hline Soybean meal & 28.00 & 26.32 & 24.64 & 22.00 \\
\hline $\mathrm{AF}$ & 0.00 & 6.00 & 12.00 & 18.00 \\
\hline Fish meal & 3.00 & 2.82 & 3.69 & 5.10 \\
\hline Whey powder & 9.00 & 5.50 & 5.50 & 5.00 \\
\hline Wheat bran & 1.00 & 1.00 & 1.00 & 1.00 \\
\hline Limestone powder & 1.30 & 1.22 & 1.14 & 0.90 \\
\hline Calcium hydroxide & 0.80 & 0.75 & 0.70 & 0.66 \\
\hline Wheat middling feed & 2.00 & 1.88 & 1.76 & 1.64 \\
\hline Rapeseed oil & 0.60 & 1.50 & 3.56 & 4.70 \\
\hline Premix & 1.00 & 1.00 & 1.00 & 1.00 \\
\hline \multicolumn{5}{|l|}{ Nutritional level } \\
\hline Digestive energy Mcal kg ${ }^{-1}$ & 3.41 & 3.33 & 3.31 & 3.26 \\
\hline Crude protein\% & 20.10 & 20.02 & 20.06 & 20.27 \\
\hline Crude fiber\% & 2.83 & 4.21 & 5.55 & 6.84 \\
\hline Lysine\% & 1.14 & 1.12 & 1.12 & 1.13 \\
\hline Methionine $\%$ + cysteine $\%$ & 0.69 & 0.67 & 0.65 & 0.65 \\
\hline Threonine\% & 0.81 & 0.81 & 0.81 & 0.82 \\
\hline Isoleucine\% & 0.87 & 0.86 & 0.86 & 0.87 \\
\hline Leucine $\%$ & 1.72 & 1.68 & 1.65 & 1.64 \\
\hline Proline\% & 0.92 & 0.92 & 0.92 & 0.91 \\
\hline Phenylalanine\% & 0.99 & 0.98 & 0.98 & 0.99 \\
\hline Tryptophan\% & 0.30 & 0.32 & 0.34 & 0.38 \\
\hline Calcium to phosphorus ratio & $2: 1$ & $2: 1$ & $2: 1$ & $2: 1$ \\
\hline
\end{tabular}

${ }^{a}$ Note: premix is available per kg of feed: VA 130-396 KIU, VD2 30-124 KIU, VE $400 \mathrm{mg}$, VK2 40-150 mg, VB1 $25 \mathrm{mg}$, VB2 75 mg, Cu 1500$7500 \mathrm{mg}$, Fe $1500-7500 \mathrm{~kg}$, Zn $1500-3700 \mathrm{~kg}$, Mn $400-3700 \mathrm{~kg}$, moisture $9 \%$, sodium chloride $6-14 \%$, total phosphorus $2 \%$, lysine $1.3 \%$, calcium $10-20 \%$, phytase 12500 U. A: $0.00 \%$ AF, B: $6.00 \% \mathrm{AF}$, C: $12.00 \% \mathrm{AF}, \mathrm{D}: \mathbf{1 8 . 0 0 \%} \mathrm{AF}$.

status. The fecal samples were recorded as normal, pasty, or watery. ${ }^{35}$ The diarrhea incidence (\%) was calculated using the method. ${ }^{36}$ The acetate, butyrate, propionate, and the total SCFAs were analyzed according to the method described. ${ }^{37}$

\subsection{Total DNA extraction}

Total DNA was extracted and quantified from $0.5 \mathrm{~g}$ of each cecal samples using a Qubit 2.0 Fluorometer (Thermo Fisher Scientific Inc. Massachusetts, USA) following the manufacturer's instructions. The amount of total DNA was estimated by the NanoPhotometer spectrophotometer (Thermo Fisher Scientific Inc. Massachusetts, USA), and the molecular size and DNA quality were measured using $1 \%$ agarose gel electrophoresis. Cecal sample preparation was performed as described. ${ }^{31}$ All DNA samples were stored at $-20^{\circ} \mathrm{C}$ until further analysis.

\subsection{S rDNA gene amplification and sequencing}

Sequencing was performed at the Annotech Genentech (Beijing Co., Ltd). Briefly, $10 \mathrm{ng}$ of DNA template was used to amplify the target region, according to the different sequencing regions, the corresponding primers: $341 \mathrm{~F}$ (5'-CCTACACGACGCTCTTCCGATCTN-3 ${ }^{\prime}$ ) and 805R (5'-GACTGGAGTTCCTTGGCACCCGAGAATTCCA- $3^{\prime}$ ) using TaKaRa's Ex
Taq enzyme to ensure amplification efficiency and accuracy. The preliminary quantification was performed using Qubit 2.0. Then, the insert size of the library was detected using Agilent 2100. The Bio-Rad CFX 96 real-time PCR instrument was used to perform the qPCR to accurately quantify the effective concentration of the library to ensure library quality. The PCR was performed as described. ${ }^{31}$ Then, the mixture of the PCR product was purified with a GeneJET Gel Extraction Kit (Thermo Fisher Scientific). The sequencing libraries were generated using a NEB Next Ultra DNA Library Prep Kit for Illumina (NEB, United States) following the manufacturer's instructions. The library quality was assessed on the Qubit@2.0 Fluorometer (Life Technologies, CA, United States) and the Agilent Bioanalyzer 2100 system. Finally, the library was sequenced on an Illumina HiSeq platform, and paired-end reads were generated.

\subsection{Sequence analysis}

For the sequences obtained by the sequencing, data filtering was completed by removing the low-quality base, Ns and joint contamination sequences to obtain a reliable target sequence for subsequent analysis. The filtered sequences were referred to as the Clean Reads. First, the corresponding Read1 and Read2 of the sequence fragments were obtained from the $5^{\prime}$ and $3^{\prime}$ ends, respectively using the sequence stitching method PEAR; ${ }^{38}$ the spliced sequence was analyzed using the QIIME software version $1.80,{ }^{39-41}$ including the extraction of operational taxonomic units (OTUs), overlap analysis, cluster analysis, Lefse analysis, phylogenetic tree construction, alpha diversity analysis, beta diversity analysis, etc. ${ }^{\mathbf{4 2}}$

The operational taxonomic units were clustered with a $97 \%$ similarity threshold. Species identification was based on the comparison of the corresponding recognition sequences of each species in the database with the OTUs. Operational taxonomic units with fewer than 2 sequences in all samples were eliminated to rule out the impact of less reliable OTUs on subsequent analysis. Change in OTUs between samples was counted and represented in heat maps. The number of unique OTUs for each group was represented on a Venn diagram. The distribution of species in each sample at each classification level was represented in histograms. According to the species annotation and abundance information of all the samples at the class, family, and genus levels, the top 25 subjects were selected based on the families, genera, and their abundance information in each sample to draw sample heat maps, and classify them according to the classification standards. Sample clustering was perform using the information available at each sample level for the easy display of results and the discovery of information to identify more species or samples that were concentrated in the sample study for the obtained species distribution data of each sample after normalization. The Bray-Curtis distance value was calculated, and each sample was clustered by a hierarchical clustering algorithm. The cluster analyzed the similarities between samples.

According to the species content of the samples, the distribution results at the five taxonomic levels comprised of the phylum, class, order, family, and genus were calculated. The information available at the phylum, class, order, family, and 
genus levels were used to draw species distribution histograms. For the identification of the dominant species in each sample, the dominance index for each species in each sample was calculated. For species similarities and diversity, the alpha diversity analysis using the Shannon diversity index, Simpson diversity, Chao 1 richness, and observed species richness were used. The principal component analysis (PCA) was performed at the genus level, ${ }^{43}$ and the results were visualized using the Statistical Analysis of Metagenomics Profiles (STAMP) version 2.13 program..$^{44}$ The linear discriminant analysis coupled with effect size (LEfSe) was performed to identify the bacterial taxa differentially represented between groups at the genus or higher taxonomy levels. ${ }^{45}$ LefSe analysis combines linear discriminant analysis with the KW rank sum test and Wilcox test to determine the effect of different microbial species in different treatment groups. Finally, the Metastats software and T-test software were used to analyzed the species abundance data between groups.

\subsection{Data analysis}

Data were analyzed using a one-way analysis of variance (ANOVA) procedure of SPSS software version 20.0 (SPSS Inc., Chicago, IL, USA). A probability value of $p<0.05$ was considered statistically significant and differences between means were noticeable. The Duncan Multiple Range Test (DMRT) was employed to ascertain the differences between the treatments.

\section{Results}

\subsection{Growth performance and piglet diarrhea}

The growth performance of piglets increased with the increase in AF supplementation, but at higher AF concentrations (D), there was a decrease in the growth performance of piglets. There was no significant difference $(p>0.05)$ in the ADG among all the treated groups. The ADFI of treatment $C$ was significantly different $(p<0.05)$ from treatment $\mathrm{A}$ and treatment $\mathrm{D}$ but not significantly different $(p>0.05)$ from treatment B. Treatment D recorded the highest $\mathrm{F}: \mathrm{G}$ with no significant difference $(p>$ $0.05)$ between the treatments. The diarrhea incidence in treatment A was significantly higher $(p<0.05)$ than the other treatment groups. Meanwhile, the lowest diarrhea incidence was recorded in treatment $\mathrm{C}$ and treatment $\mathrm{D}$ (Table 2).

\subsection{Cecal short-chain fatty acids production in pigs}

As shown in Table 3, the supplementation of AF increased the acetate and propionate levels. The highest acetate and propionate levels were observed in treatment $\mathrm{B}$, but there was no significant difference $(p>0.05)$ between the treatment groups. The butyrate concentration increased with the increase in $\mathrm{AF}$ concentration. There was a significant increase $(p<0.05)$ in butyrate production in treatments $\mathrm{C}$ and $\mathrm{D}$ in comparison with treatments A and B. The highest total SCFAs was observed in treatment B, but there was no significant difference $(p>0.05)$ between the groups.

\subsection{Sequence description analysis}

The high throughput pyrosequencing of the cecal samples produced 65590 overlapping pair-end reads from the 16 samples; 61618 clean reads were obtained representing 93.94\% of reads to be subjected to further analysis. The clean reads were combined to tag bases. About 30809 total reads were obtained with a mean of 460.32 tags per sample, and all reads were assembled. 27867 normalized tags of the OTUs were obtained with total aligned tags of 25094 . The aligned rate per sample was $90.05 \%$, and a total number of OTUs of 210 was obtained by clustering the 30809 tags at $97 \%$ similarity (Table 4). The overlap of OTUs between groups was represented in a Venn diagram (Fig. 1). The Venn diagram estimates the overlapping OTUs composition and species overlap between the treatment groups. Fig. 1 showed that 334 OTUs were shared among all sample groups. Higher species specificity was observed in treatment B with 10 OTUs, while treatment A, C, and D have OTUs specificity of 4 each. The shared OTUs between the groups were 37 and between treatments $\mathrm{B}, \mathrm{C}$, and D; 7 OTUs were shared between treatments A, B, and D; 10 OTUs were shared between treatments A, B, and C. 18 OTUs were shared between A, C, and D. The shared OTUs between two sample treatments were 18 between treatment $\mathrm{C}$ and $\mathrm{D}, 1$ OTU between treatment $\mathrm{A}$ and C, and 1 OTU between treatment A and D, 3 OTUs between $\mathrm{B}$ and C, and 8 OTUs between B and D (Fig. 1).

\subsection{Gut microbiota richness and diversity}

There was no significant difference $(p>0.05)$ in species richness, as represented by the Chao 1 and Observed_species values in the treatments (Table 5 and Fig. 2). The species richness increased with the increase in the supplementation of AF, reaching a plateau and then decreasing as the $\mathrm{AF}$ content increased (treatment D). The highest Chao 1 and Observed_species values were recorded in treatment $\mathrm{B}$ and the lowest in the control group (A). There was no significant difference ( $p>$ 0.05 ) in species diversity in all treatment groups. The highest Shannon diversity index value was found in treatment $\mathrm{B}$, and

Table 2 Effects of AF on the growth performance and diarrhea incidence in weaned piglets (kg)

\begin{tabular}{lllll}
\hline Items & $\mathrm{A}$ & $\mathrm{B}$ & $\mathrm{C}$ & $\mathrm{D}$ \\
\hline ADG & $1.16 \pm 0.17^{\mathrm{a}}$ & $1.25 \pm 0.19^{\mathrm{a}}$ & $1.32 \pm 0.16^{\mathrm{a}}$ & $1.04 \pm 0.23^{\mathrm{a}}$ \\
ADFI & $2.02 \pm 0.55^{\mathrm{b}}$ & $2.13 \pm 0.22^{\mathrm{a}, \mathrm{b}}$ & $2.22 \pm 0.19^{\mathrm{a}}$ & $1.89 \pm 0.06^{\mathrm{b}, \mathrm{c}}$ \\
F : G & $1.74 \pm 0.20$ & $1.70 \pm 0.23$ & $1.68 \pm 0.38$ & $1.82 \pm 0.32$ \\
Diarrhea (\%) & $6.94 \pm 0.12^{\mathrm{a}}$ & $4.17 \pm 0.25^{\mathrm{b}}$ & $0.37 \pm 0.21^{\mathrm{c}}$ & $0.35 \pm 0.11^{\mathrm{c}}$
\end{tabular}

${ }^{a}$ Note: A: $0.00 \%$ AF, B: $6.00 \%$ AF, C: $12.00 \%$ AF, D: $18.00 \%$ AF. Different alphabets in the same column denote a significant difference, and the same alphabets or no alphabets means that they were not significantly different. A significant difference was observed at $p<0.05$. 
Table 3 Effect of AF supplementation on short-chain fatty acids in the cecum of piglets $\left(\mathrm{mmol} \mathrm{L}^{-1}\right)$

\begin{tabular}{|c|c|c|c|c|}
\hline Items & A & B & $\mathrm{C}$ & $\mathrm{D}$ \\
\hline Acetate & $35.154 \pm 5.93^{\mathrm{a}}$ & $43.27 \pm 8.97^{\mathrm{a}}$ & $39.84 \pm 2.11^{\mathrm{a}}$ & $38.17 \pm 5.82^{\mathrm{a}}$ \\
\hline Propionate & $29.92 \pm 1.41^{\mathrm{a}}$ & $32.78 \pm 1.73^{\mathrm{a}}$ & $31.96 \pm 4.03^{\mathrm{a}}$ & $31.72 \pm 4.30^{\mathrm{a}}$ \\
\hline Butyrate & $21.56 \pm 1.66^{\mathrm{b}}$ & $22.13 \pm 2.58^{b}$ & $25.21 \pm 2.35^{\mathrm{a}}$ & $25.78 \pm 7.17^{\mathrm{a}}$ \\
\hline Total SCFAs & $86.63 \pm 6.75$ & $98.18 \pm 10.01$ & $97.01 \pm 4.73$ & $95.67 \pm 3.18$ \\
\hline
\end{tabular}

${ }^{a}$ Note: A: $0.00 \%$ AF diet, B: $6.00 \%$ AF diet, C: $12.00 \%$ AF diet, D: $18.00 \%$ AF diet. Different alphabets in the same column denote a significant difference, and the same alphabets or no alphabets means that they were not significantly different. A significant difference was observed at $p<0.05$.

the lowest Shannon diversity index was observed in treatment D. The Simpson diversity value increased with the increasing AF content. The highest Simpson diversity index was observed in treatment D and the lowest in treatment A. The PCA and nonmetric multi-dimensional scale method (NMDS) reflects the differences between samples in the two-dimensional coordinate map (Fig. 3B). The more similar the composition of the microflora between the samples, the closer the distance representing their coordinate points in the PCA plot. The contribution rates of $\mathrm{PC} 1$ and $\mathrm{PC} 2$ in Fig. $3 \mathrm{~A}$ were $45.73 \%$ and $19.71 \%$, respectively, with a cumulative contribution rate of $65.44 \%$. On the PC2 axis, treatments A and B were distributed towards the positive direction, while treatments $\mathrm{C}$ and $\mathrm{D}$ were mainly distributed towards the negative direction. The supplementation of AF increased the microbial community distribution in each sample, but the microbial composition between the treatments was similar. The closer the PCA plot in the

Table 4 Data sequencing statistical analysis involving data filtering statistics, sequencing splicing, and OTU statistics ${ }^{a}$

Sample Statistics

\section{Filtering statistical}

Raw reads

Raw bases (bp)

Clean reads

Clean reads rate $(\%)$

Clean bases (bp)

Low-quality reads

Low-quality reads rate (\%)

Ns reads

Ns reads rate $(\%)$

Adapter polluted reads

Adapter polluted reads rate $(\%)$

Raw Q30 bases rate (\%)

Clean Q30 bases rate (\%)

\section{Sequence splicing}

Total reads

Total assembled reads

Total assembled rate (\%)

Average assembled length

Std assembled length

65590

16397500

61618

93.94

18177310

3972

6.06

0.0

0.0

0.0

0.0

80.63

82.32

\section{OTUs summary statistics}

Normalized tags

Total aligned tags

Aligned rate (\%)

Total OTUs number

30809

30809

100.0

460.32

9.39

${ }^{a}$ Notes: Table 3 represents the data sequencing analysis involving data filtering statistics, sequencing splicing, and OTUs statistics. distribution principal components in Fig. 3A, the more similar the community composition of the samples.

\subsection{Cecal microbiota composition}

The effects of AF on piglets cecal microbial composition were observed at different taxonomic levels. With regard to the phyla, a total of 15 phyla were identified in each sample. There was no significant difference $(p>0.05)$ in the phylum microbial composition between the treatment groups and the control. Bacteroidetes and Firmicutes were the main dominant phyla, accounting for about $98 \%$ of all reads (Fig. 4A). The Bacteroidetes phylum was significantly higher $(p<0.05)$ in all treatment groups compared with the other bacterial phyla. The Bacteroidetes was the most dominant phylum; the relative abundance of the Bacteroidetes decreased slightly upon the addition of $\mathrm{AF}$ and then increased at a steady rate as the $\mathrm{AF}$ concentration increased. The Firmicutes concentration increased with the increasing AF concentration; at the highest AF level, there was a decreasing trend observed (Fig. 4A).

At the class level, a total of 23 bacterial classes were observed across all treatment groups (Fig. 4B). Our results indicated that Bacteroidia, Bacilli, Clostridia, and Negativicutes were the dominant bacteria classes. The relative abundance of Bacteroidia was significantly different $(p<0.05)$ in all treated groups compared to the other bacteria classes. The Bacilli significantly increased $(p<0.05)$ in treatment $\mathrm{C}$ compared to the other treated groups. The Negativicutes class was not significantly affected by the addition of AF in the diet. According to the increasing order of the dominant bacteria classes, Bacteroidia $>$ Bacilli $>$ Clostridia $>$ Negativicutes, accounting for $95 \%$ of the total reads (Fig. 4B).

A total of 26 bacteria orders were identified in each treatment (Fig. 4C). The cecal microbial composition was dominated by the following orders: Bacteroidales (65\%), Lactobacillales (15\%), Clostridiales (10\%), and Selenomonadales (5\%) (Fig. 4C). The Bacteroidales was significantly different $(p<0.05)$ in all the treatment. The Bacteroidales decreased as the concentration of $\mathrm{AF}$ increased. The Clostridiales increased with an increase in AF levels. The Selenomonadales order increased with the increase in AF supplementation. The Lactobacillales levels maintained a steady state level with the increase in $\mathrm{AF}$; at the highest $\mathrm{AF}$ level (D), there was a reduction in the relative abundance of Lactobacillales.

At the family level, 26 families were characterized within each treatment group (Fig. 4D). Bacteroidaceae, Lactobacillaceae, Streptococcaceae, and Veillonellaceae were the dominant bacteria 


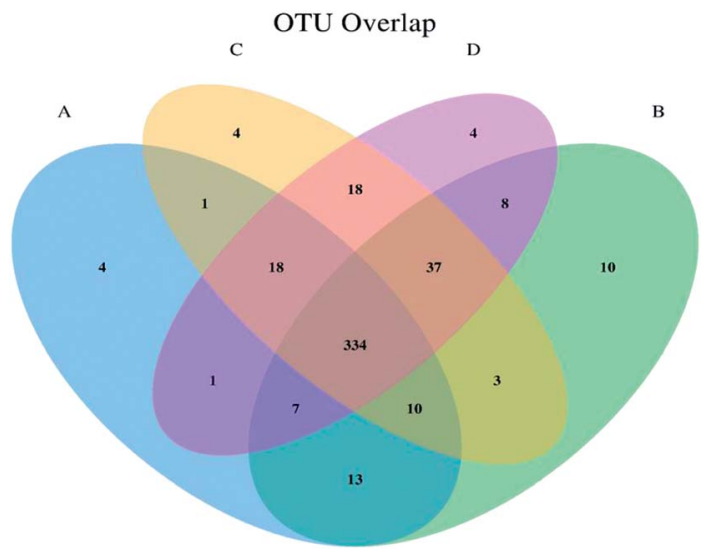

Fig. 1 Venn diagram showing the overlap between groups. The Venn diagram represents the OTUs similarities and dissimilarities shared between samples. The letters $A, B, C$, and $D$ represent the treatment groups, and the numbers represent the OTUs.

families. The supplementation of AF had no significant effect on the Bacteroidaceae and Streptococcaceae families in each treatment group. There was a slight increase in the relative abundance of Lactobacillaceae and Veillonellaceae as the AF content increased (Fig. 4D).

At the genera level, 26 genera were characterized (Fig. 4E). Prevotella 2, Prevotella 9, uncultured, and Streptococcus were the dominant species in all treatments (Fig. 4E). The relative abundance of Prevotella 9 was significantly dominant $(p<0.05)$ in all treatment groups compared with the other bacterial genera. The supplementation of AF in the diet of piglets slightly increased the relative abundance of Prevotella 2 and Prevotella 9 at higher concentrations of $\mathrm{AF}$ (D). AF treatment has no effect on the Uncultured and the Streptococcus bacterial populations.

\subsection{Cecal microbial network}

Fig. 5A showed that the bacterial members with significantly high abundance mainly belonged to the Bacteroidia, Clostridia, and Negativicutes classes. The bacterial taxa with significantly high population abundance were dominated by the Bacteroidales, Clostridiales, and Selenomonadales orders in the treatment groups. The bacterial taxa with significantly greater population were dominated by the Prevotellaceae family (Fig. 5A). From Fig. 5B, the $R$-value was -0.083 and the $p$-value was 0.662 , implying that the $R$-value was closer to 0 and the $p$ - value was greater than 0.05 . This means that there was no significant difference $(p>0.05)$ between and within the treatment groups.

From Fig. 5C, 11 bacterial taxa were shown to be specific to treatment $\mathrm{D}$ and, hence, influence the community structure. A total of 10 bacterial taxa were specific to treatment C; these bacterial taxa were specific and influenced the community structural composition in treatment C. 2 bacterial taxa were specific and influenced the community structural composition in treatment B. In treatment A, 5 bacterial taxa were specific and influenced the bacterial composition. Fig. 5D showed that the bacterial members with significantly high abundance mainly belonged to the $f_{-}$Bacteroidales $R F 16$ group and $f_{-}$Helicobacteraceae families in treatment $\mathrm{D}$. In addition, the bacterial taxa with significantly greater population were dominated by the $f_{-}$family, $f_{-}$family III, and O_campylobacterales order in treatment A. In treatment B, the relative abundance of the bacterial taxa with significantly greater population were the $O_{-}$campylobacterales and C_Epsilonproteobacteria classes. There were three families with significantly dominant bacteria population, $f_{-}$Clostridiaceae, $f_{-}$Clostridiaceae 1 , and $f_{-}$Peptostreptococceae, in treatment group $\mathrm{C}$.

\section{Discussion}

Early weaning is a normal husbandry practice in most global swine production. ${ }^{46}$ Weaning is a harsh condition that is mostly associated with growth disorders and health effects in piglets. Piglets often exhibit a reduction in feed consumption and low nutrient metabolism due to their immature digestive systems, stress as a result of the separation from the dam and littermates, and the sudden change from the easily digestible sow's milk to a less digestible solid feed ${ }^{26}$ These piglets have limited susceptibility to diseases and infections from enteric bacteria in addition to frequent diarrhea, stunted growth, low nutrient utilization efficiency, and even mortality. ${ }^{31,47}$ Due to the reduction in small intestinal composition, limited immunological response, and other protections derived from the maternal milk, piglets are more susceptible to diseases and infections by enteric bacteria like E. coli and Salmonella ${ }^{46,47}$ There have been reports that the adaptation of piglets to a weaning diet takes more than 14 days. ${ }^{48}$ The composition and functions of the gut microbiota can be influenced by dietary nutrient

Table 5 Effects of AF on microbial species richness and diversity ${ }^{a}$

\begin{tabular}{llllll}
\hline Items & A & B & C & D \\
\hline
\end{tabular}

\section{Richness index}

Chao 1

Observed_species

$$
312.39 \pm 36.59
$$

$246.1 \pm 35.91$

$5.18 \pm 0.12$

$0.89 \pm 0.06$
$318.23 \pm 56.84$

$288.77 \pm 53.18$

$5.91 \pm 0.30$
$0.93 \pm 0.05$
$316.77 \pm 28.87$

$277.3 \pm 39.57$

$5.33 \pm 0.62$

$0.92 \pm 0.03$
D

$311.29 \pm 5.00$

$263.67 \pm 30.20$

\section{Diversity index}

Simpson

${ }^{a}$ Notes: Table 4 represents the effects of AF on microbial richness and diversity. The microbial richness was represented with the Chao 1 index and the Observed_species index, and the microbial diversity was represented with the Shannon and Simpson diversity indices. The letters A, B, C and D represent the treatment groups. There was no significant difference $(p>0.05)$ in the microbial richness and diversity. 
Shannon

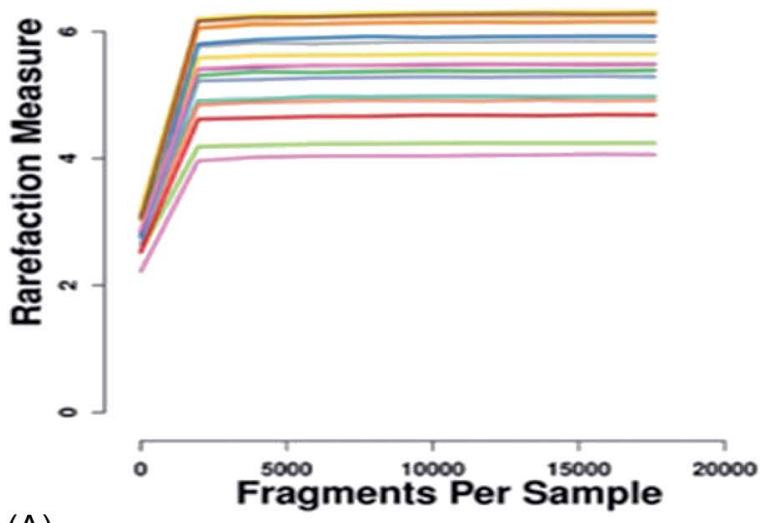

(A)

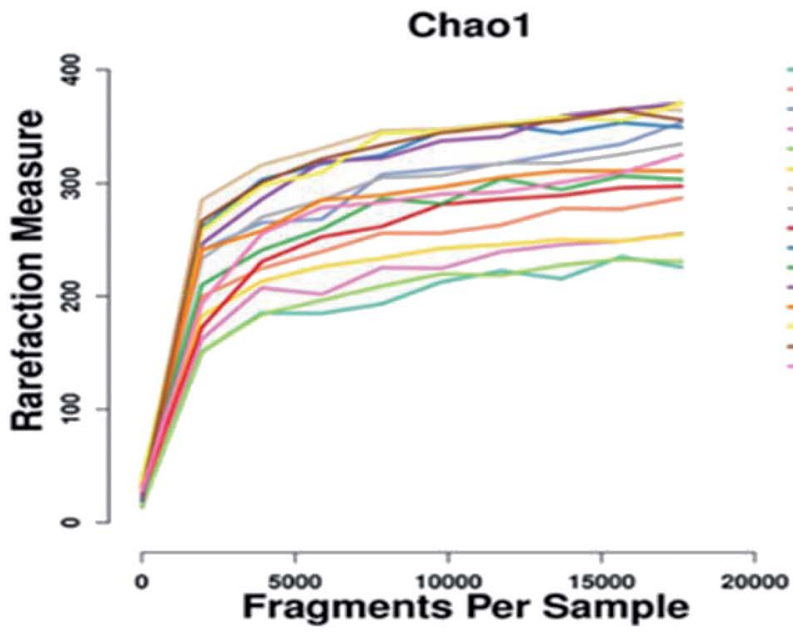

(B)

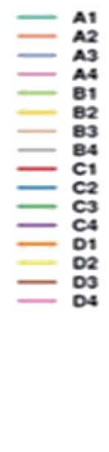

(C)

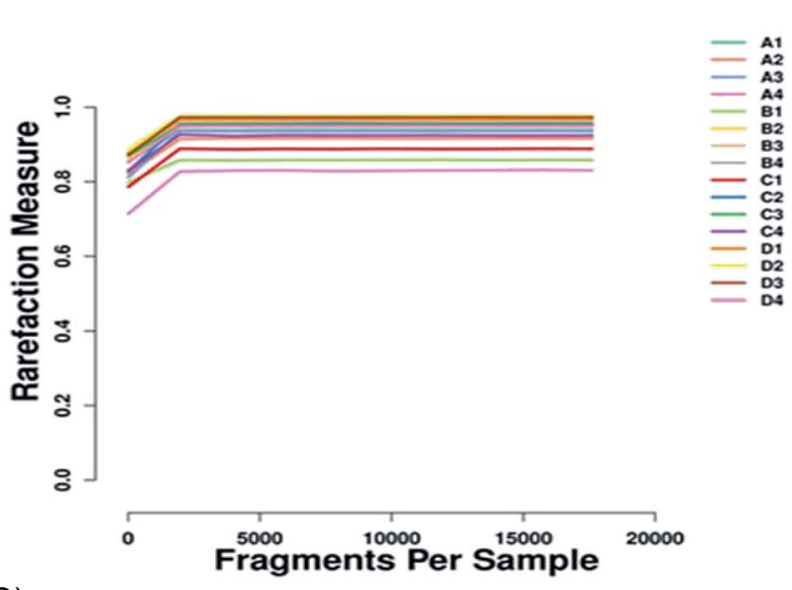

Simpson

Observed_species

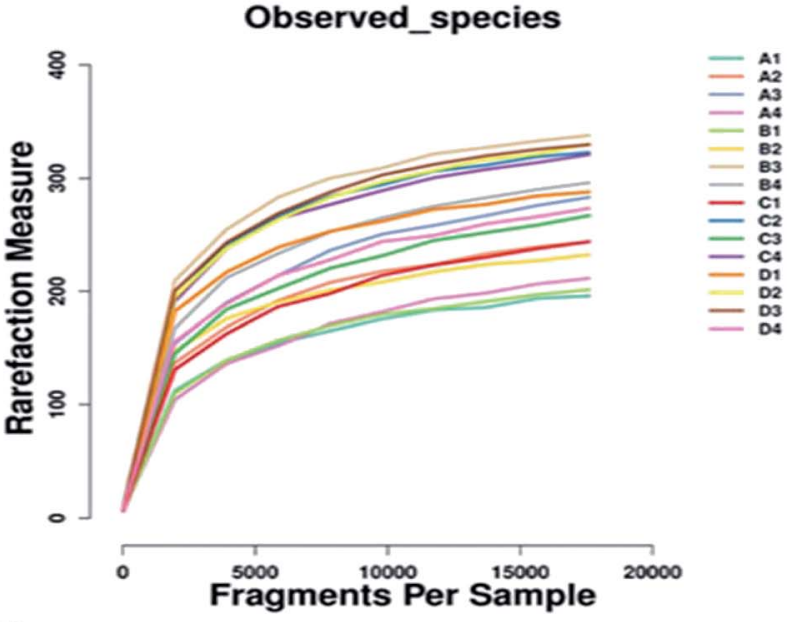

(D)

Fig. 2 The alpha indices estimating species richness and diversity between groups. (A) The Shannon diversity index, (B) the Chao 1 index, (C) the Simpson diversity index, and (D) Observed_species.

supplementation. ${ }^{49}$ Therefore, the objective of this study was to investigate the prebiotic effects of $\mathrm{AF}$ supplementation to the diet of early weaning pigs on cecum microbial composition and function, piglet diarrhea, and SCFAs production.

In global pig farming, several dietary-fiber-based feeds have been used to increase the nutrient digestibility and growth performance of pigs ${ }^{50}$ and improve their gut microbial composition. However, the effects of dietary fiber supplementation on growth performance in pigs are inconsistent. ${ }^{36}$ Many studies have indicated that high dietary fiber supplementation increased growth, ${ }^{51-53}$ while others observed no impact on growth performance. ${ }^{29,30,54}$ The changes in the gut milieus and nutrient digestion can improve the gut microbial composition, production performance, and health of pigs. Previous studies in our group ${ }^{28,46}$ reported that Astragalus membranaceus fiber supplemented in a corn-soybean-based diet significantly increased the growth performance of piglets. Similarly, the findings of this study showed that the supplementation of AF increased the growth performance of piglets, but at higher AF concentrations, there was a decrease in growth performance. This result implies that increasing the AF content of the diet may improve the gut milieus, increase growth, and consequently improve the gut microbial composition of piglets, but at higher AF levels, there may be a detrimental effect on the growth performance of piglets. ${ }^{26}$ The findings of this current study were consistent with our previous investigation ${ }^{31,46}$ and that of other reports..$^{51,55}$ The reason for the observed decreased in growth performance of piglets at high AF levels may be that piglets cannot metabolize high-fiber diets due to their immature digestive tracts and the low microbial composition. ${ }^{26}$ In contrast to our findings, ${ }^{56,57}$ no effects were reported on the ADFI, ADG, or FCR after supplementing dietary corn bran to the diet of weaned piglets. Our results further elucidated that the supplementation of AF to the diet of piglets significantly decreased diarrhea incidence in comparison with the control. This observation was consistent with the results. ${ }^{3,16,31,32,58}$ This observation may be due to the rich bioactive compound composition of $\mathrm{AF}$, which may exert antiinflammatory and anti-microbial effects on the gut of weaning piglets thereby reducing the population of enteric bacteria in the gut. Therefore, decreasing the diarrhea incidence in pigs. 


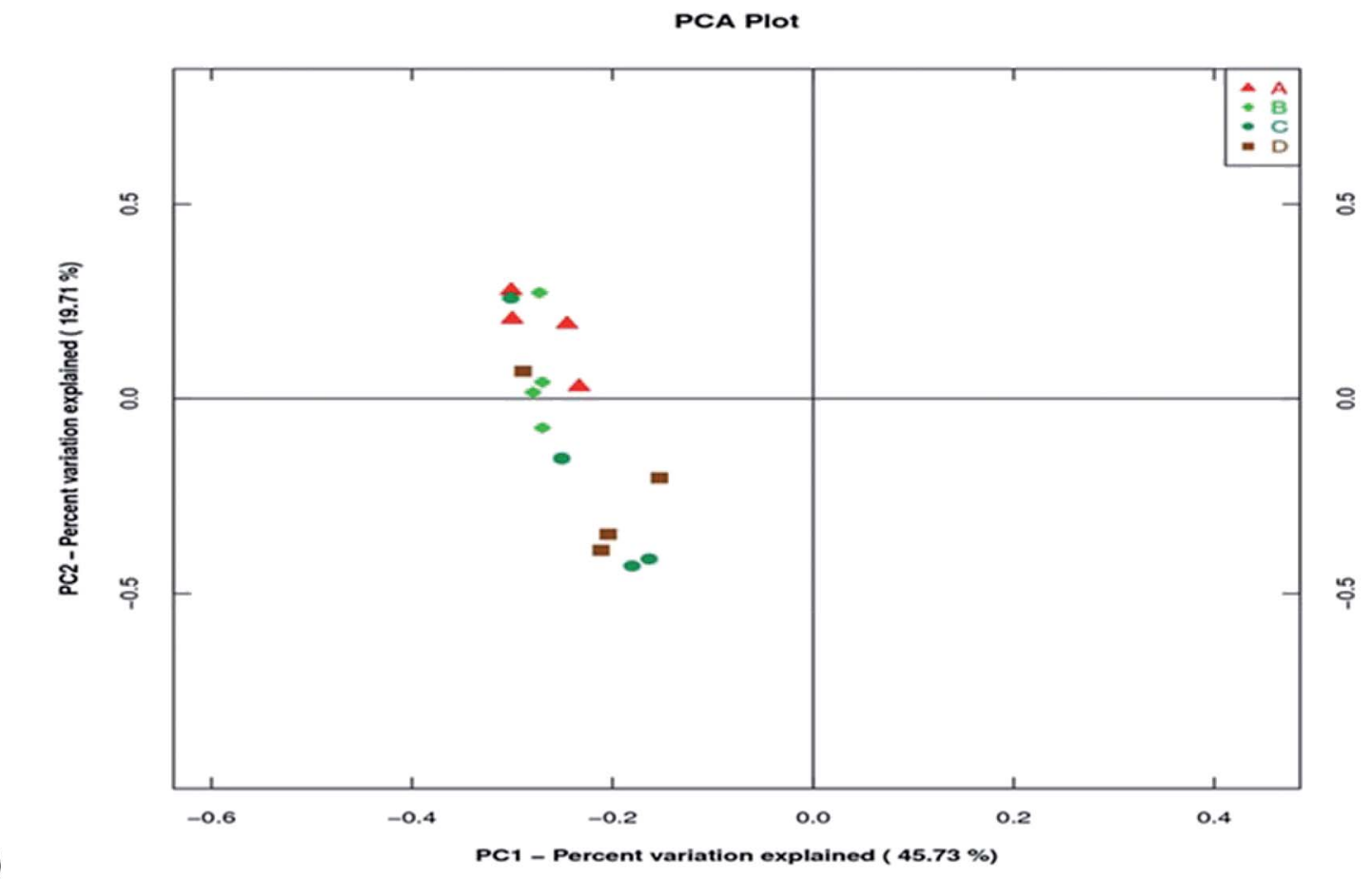

(A)

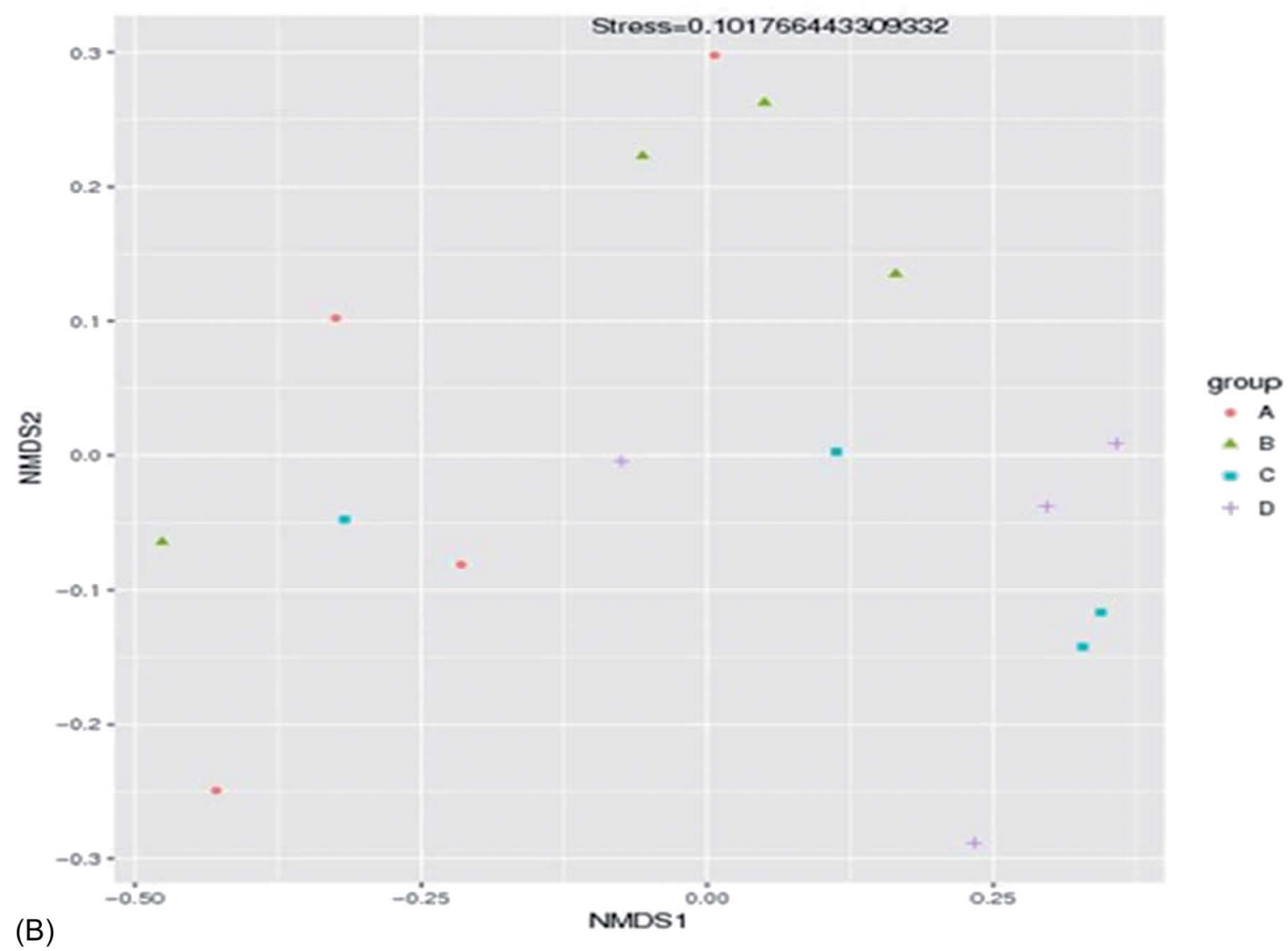

Fig. 3 The PCA (A) and NMDS (B) of species similarity and dissimilarity between groups.

We further evaluated the effects of AF on SCFAs production in pigs. Short-chain fatty acids, also known as volatile fatty acids (VFA), mainly include acetic acid, propionic acid, and butyric acid, which are involved in organ metabolic processes in the body and exert their respective functions. The synthesized acetic acid enters the blood and participates in the metabolic processes of the liver, heart, spleen, and the brain..$^{59}$ Propionic acid and butyric acid not only provide energy for liver 
Phylum Distribution

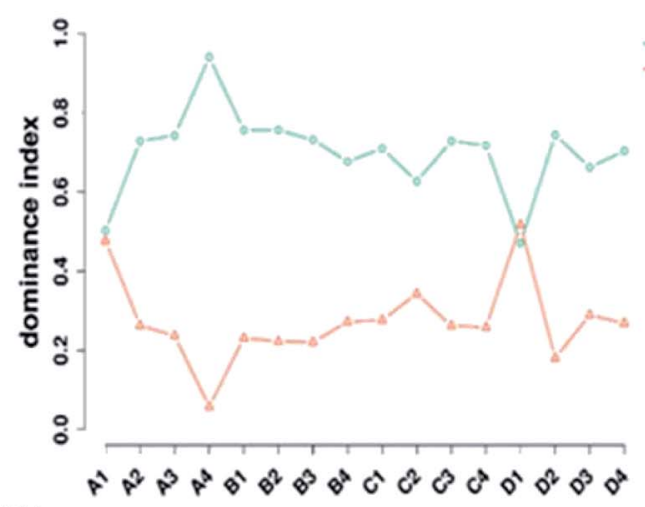

(A)

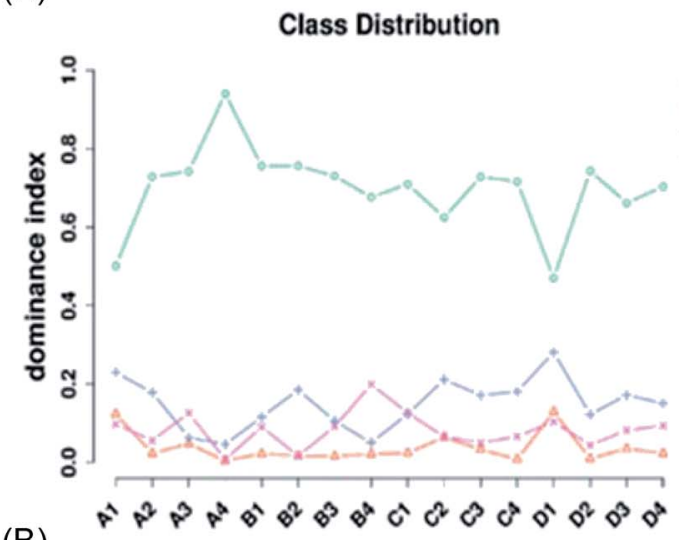

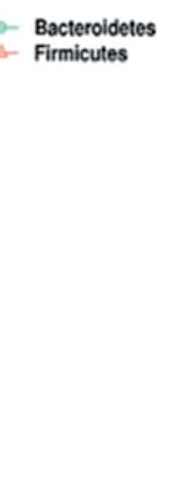

$(\mathrm{C})$

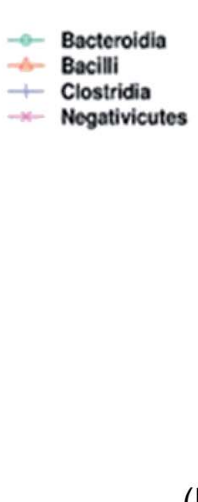

Order Distribution

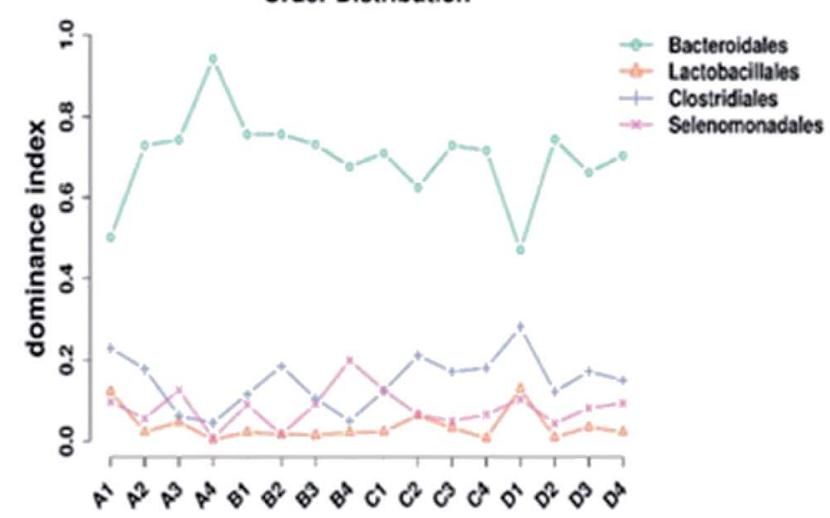

(C)

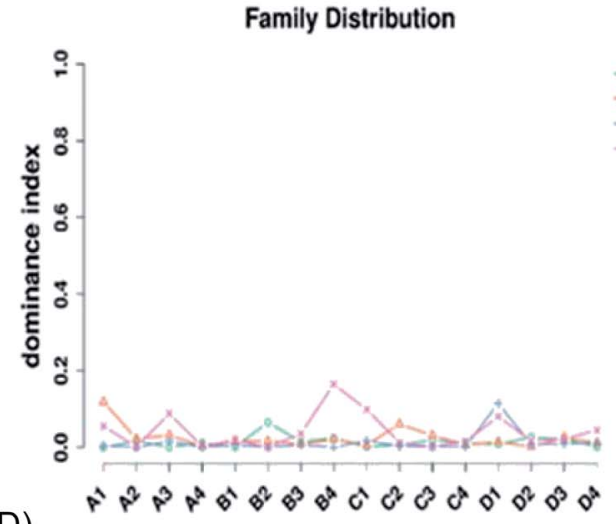

Gonus Distribution

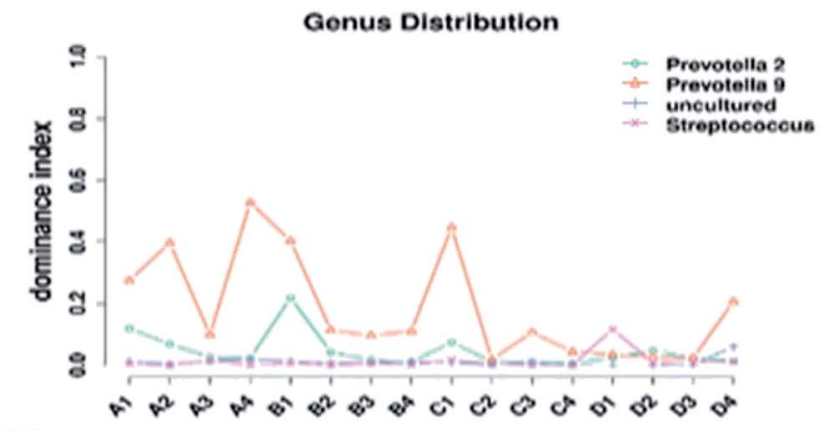

(E)

Fig. 4 Line graph representing the distribution of the dominant bacteria species in the taxa. (A) Phylum distribution, (B) class distribution, (C) order distribution, (D) family distribution, (E) genus distribution.

metabolism but also inhibit cholesterol synthesis; these SCFAs regulate the expression of genes and maintain stability within the intestinal tract. From the results of this experiment, the contents of acetate, propionate, and butyrate in the cecum of the AF-fed groups were higher than that of the control group. This study was consistent with the findings. ${ }^{15,31}$ Also, Gidenne et al. found that an increase in fiber levels can increase the concentration of VFAs in the cecum and concluded that a highfiber diet can be fermented in the cecum to produce SCFAs to provide energy for the body. ${ }^{60}$ However, acetate and propionate content decreased at higher dietary AF levels, indicating that excess fiber may decrease the microbial activities in the cecum. The butyrate levels significantly increased with the increase in dietary AF levels. In contrast to our findings, Zhang et al. reported lower ileal VFA concentrations in pigs fed corn-based diets. ${ }^{61}$ In addition, Freire et al. reported low VFA production in pigs after changing the diet from sugar-beet pulp to soybean hulls. ${ }^{62}$ Conversely, insoluble dietary fiber can decrease the transit time, leading to a reduction in nutrient absorption in the small intestine. ${ }^{52,56}$ Short-chain fatty acids such as propionic and butyric acids were reported to activate the GPR43 on enteroendocrine L-cells to increase gut hormonal synthesis, such as PYY and GLP1, which play vital roles in appetite regulation through the gut-brain axis. ${ }^{56}$ High fiber intake increased the synthesis of PYY and GLP in addition to inhibiting energy consumption and increase satiety. ${ }^{56,63}$ Therefore, the increase in the synthesis of appetite-stimulating hormones through the consumption of dietary fiber coupled with the nutrient-rich $\mathrm{AF}$ 

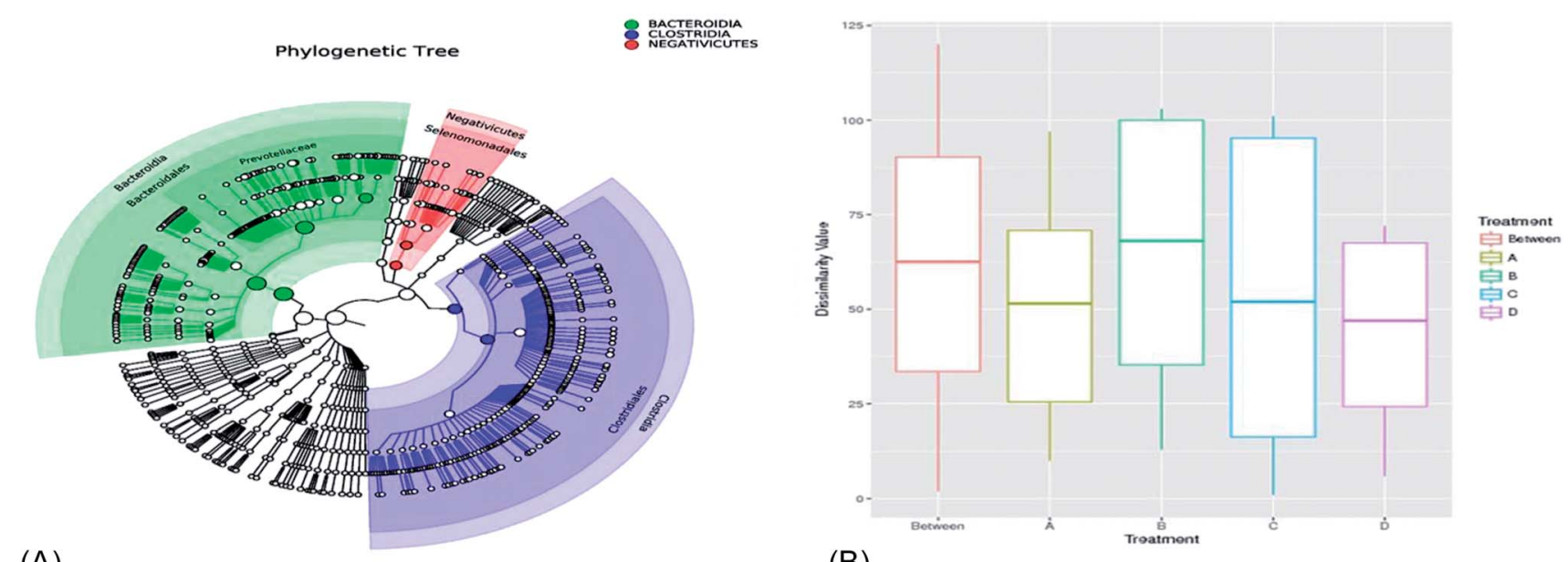

(A)

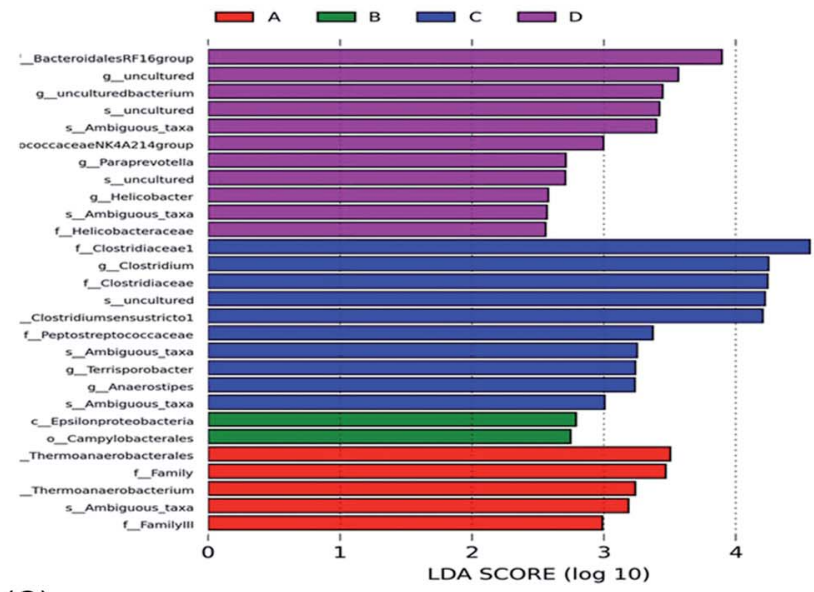

(C)
(B)

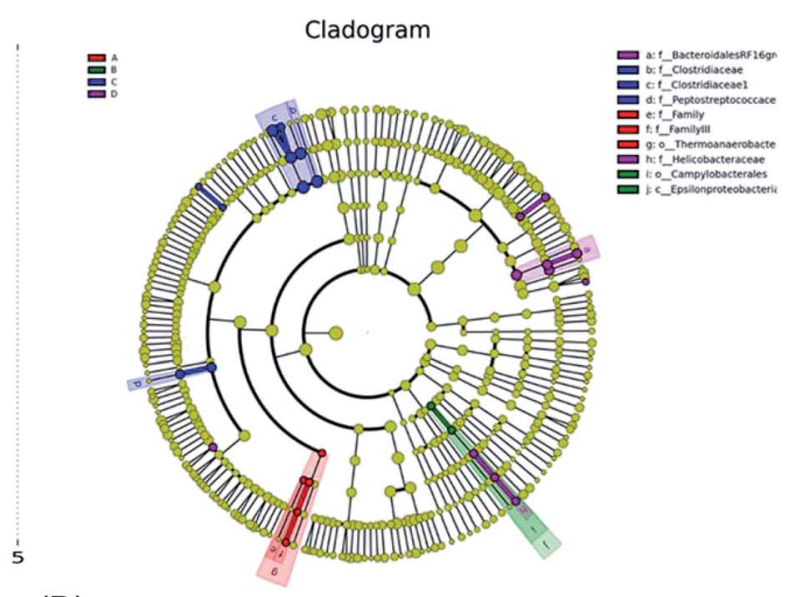

(D)

Fig. 5 (A) Species evolution tree, (B) anosim analysis of taxa similarity, (C) LDA value distribution histogram example of the LefSe evolution branch diagram; (D) the cladogram of LEfSe demonstrates the taxonomic profiling for the distinct bacteria with significant higher abundances. The circles from inwards to outwards represent the different levels of bacteria members ranged from phylum to genus. The results were analyzed by the wilcoxon rank-sum test and are presented as the mean relative abundance with one asterisk meaning $p<0.05$. LEfSe, linear discriminant analysis effect size.

diet may be a vital insight into the observed increase in the growth performance and synthesis of energy metabolites. Moreover, the mechanism of action may be due to the positive impact of AF on the cecal microbial composition of piglets.

The gut contains large volumes of microorganisms that play vital roles in both human and animal well-being. High throughput 16S-rDNA sequencing was used in this study to evaluate the abundance and diversity of the cecum microbial composition in piglets. The supplementation of $\mathrm{AF}$ regulates the cecum microbial richness and diversity by enhancing the production of SCFAs through the fermentation of fibers. The knowledge of the diversity of the gut microbial composition during the production period in animals is very important. ${ }^{64}$ The microbial richness and diversity analysis indicates that there was no significant difference in species richness and diversity between the gut microbiota in all treatment groups. However, AF supplementation increased species richness in treatment $\mathrm{B}$ and decreased as the fiber content of the diet increased. The Shannon diversity first increased and later decreases as the AF content of the diet reached higher levels, while the Simpson diversity increased with the increase in AF levels. Our findings were consistent with the results of Che et al., who observed an increase in species richness and diversity after incorporating Astragalus membranaceus fiber in the diet of piglets. ${ }^{31}$ In addition, Ran et al. reported an increase in the species richness and observed OTUs after supplementing thymol and carvacrol in the diet of hybrid tilapia. ${ }^{65}$ In contrast with our study was the findings of Liu et al., who reported that there were no differences in the species diversity and richness in the colonic microbial composition of pigs. ${ }^{56}$ In the cecal gut microbiota of piglets, Bacteroidetes (72\%) and Firmicutes (26\%) were the dominant bacterial phyla. Consistent with our findings was the study by Lamendella et al., who reported that Bacteroidetes and Firmicutes were the dominant bacteria in the pig cecum. ${ }^{66}$ Similarly, other studies ${ }^{31,33}$ reported that the Bacteroidetes and Firmicutes were the dominant bacterial phyla in the cecum of piglets. In addition, recent researchers reported that Firmicutes and Bacteroidetes were the predominant phyla in the gut microbiota of pigs. ${ }^{33,56,67-69}$ We observed that the relative abundance of Bacteroidetes was higher than the relative 
abundance of Firmicutes. Similarly, Klein-Jöbstl et al. reported that Bacteroidetes was the highest relative abundant phylum in the gut of calves. ${ }^{64}$ In contrast, Mateos et al. and Kim et al. reported that Firmicutes was the highest relatively abundant phylum in cattle. ${ }^{70,71}$ The supplementation of AF in the diet increased upon increasing the relative abundance of Firmicutes, while the Bacteroidetes population decreased upon the addition of AF. The changes in these two microbial strains may affect the metabolism and functions of the gut microbiota. Similarly, Li et al. reported that the addition of essential oils increased the Firmicutes phylum population while decreasing the relative abundance of the Bacteroidetes phylum. ${ }^{3}$ In contrast to this observation, Liu. et al. reported a decrease in the Firmicutes phylum population and an increase in the Bacteroidetes phylum after feeding a corn-based diet to piglets. ${ }^{56}$ The reason for the high abundances of Bacteroidetes was reported in previous studies $^{72,73}$ that the Bacteroidetes were the main degraders of complex polysaccharides in the gut of pigs due to their ability to encode genes for glycoside hydrolases such as $\beta$-xylosidases, endo-1,4- $\beta$-xylanases, $\alpha$ - $N$-arabinofuranosidases, and polysaccharide lyases compared with the Firmicutes phylum or other bacterial phyla in the gastrointestinal tract. Meanwhile, recent studies have indicated that the Firmicutes to Bacteroidetes ratio is of significance in human gut microbial composition and functions in regards to energy metabolism and health. ${ }^{74}$ For example, Turnbaugh et al. reported that a higher Firmicutes to Bacteroidetes ratio improved the digestibility of nutrients in pigs. ${ }^{75}$ Related studies indicated that the Bacteroidetes in the cecum can encode glycosidic hydrolysis and polysaccharide cleavage genes that promote polysaccharide degradation, ${ }^{76}$ while Firmicutes play a role in digesting carbohydrates, ${ }^{72}$ which can extract more energy through the fermentation of fiber to synthesize more SCFAs. The relative abundance of the Firmicutes in groups $\mathrm{B}$ and group $\mathrm{C}$ was slightly higher than that in the control group. Consistently, this indicating that dietary fiber can promote the growth of the Firmicutes phylum.

\section{Conclusions}

In summary, piglets were fed with different concentrations of dietary AF for 48 days. The prebiotic effect of dietary AF was investigated on the diarrhea incidence, SCFAs production, and microbiota composition in weaning piglets. Dietary inclusion of $\mathrm{AF}$ in the diet of weaning piglets increased their growth performance and decreased diarrhea occurrences. In addition, the added AF content promoted the composition and diversity of the gut microbiota of piglets. However, the functions of the different microbiota inhabiting in the cecum of piglets were not estimated. Therefore, further research is recommended to elucidate the functional roles of all the microbial species involved in growth promotion, SCFAs synthesis, nutrient digestion, immunity, and energy metabolism.

\section{Conflicts of interest}

The authors declare no conflicts of interest.

\section{Acknowledgements}

This work was financially supported by the National Key Research and Development Program of China (2017YFD0502104) and the Scientific Project of Jilin Province (20170309003NY \& 20180101023JC).

\section{Notes and references}

1 S. Adams, D. Che, G. Qin, H. Rui, C. T. Sello and J. Hailong, Interactions of Dietary Fibre with Nutritional Components on Gut Microbial Composition, Function and Health in Monogastrics, Curr. Protein Pept. Sci., 2018a, 19, 1011-1023.

2 D. E. King, A. G. Mainous and C. A. Lambourne, Trends in dietary fiber intake in the United States, 1999-2008, J. Acad. Nutr. Diet., 2012, 112, 642-648.

3 Y. Li, X. Fu, X. Ma, S. Geng, X. Jiang, Q. Huang, C. Hu and X. Han, Intestinal Microbiome-Metabolome Responses to Essential Oils in Piglets, Front. Microbiol., 2018, 9, 1988.

4 W. S. Garrett, J. I. Gordon and L. H. Glimcher, Homeostasis and inflammation in the intestine, Cell, 2010, 140, 859-870.

5 W. Strober, I. Fuss and P. Mannon, The fundamental basis of inflammatory bowel disease, J. Clin. Invest., 2007, 117, 514521.

6 E. M. Ha, The impact of gut microbiota in human health and diseases: implication for therapeutic potential, Biomol. Ther., 2011, 19, 155-173.

7 L. X. Yu, X. Liu, W. Boge and X. P. Liu, Genome-wide association study identifies loci for salt tolerance during germination in autotetraploid alfalfa (Medicago sativa L.) using genotyping-by-sequencing, Front. Plant Sci., 2016a, 7, 956.

8 C. Fu, T. Hernandez, C. Zhou and Z. Y. Wang, Alfalfa (Medicago sativa L.), in Agrobacterium Protocols, Springer, New York, 2015, pp. 213-221.

9 K. E. B. Knudsen, Carbohydrate and lignin contents of plant materials used in animal feeding, Anim. Feed Sci. Technol., 1997, 67, 319-338.

10 H. Chen, X. Mao, J. He, B. Yu, Z. Huang, J. Yu, P. Zheng and D. Chen, Dietary fibre affects intestinal mucosal barrier function and regulates intestinal bacteria in weaning piglets, Br. J. Nutr., 2013, 110, 1837-1848.

11 National Research Council, Nutrient Requirements of Poultry, National Academy Press, Washington, DC, USA, 9th revised edn, 1994.

12 S. Adams, X. Kong, D. Che, G. Qin and H. Jiang, Effects of dietary supplementation of alfalfa (Medicago sativa) fibre on the blood biochemistry, nitrogen metabolism, and intestinal morphometry in weaning piglets, Appl. Ecol. Environ. Res., 2019c, 17, 2275-2295, DOI: 10.15666/aeer/ 1702_22752295.

13 J. Wang, C. Qin, T. He, K. Qiu, W. Sun, X. Zhang and J. Yin, Alfalfa-containing diets alter luminal microbiota structure and short chain fatty acid sensing in the caecal mucosa of pigs, J. Anim. Sci. Biotechnol., 2018, 9, 11. 
14 B. X. Xie, Effects of Flavonoids on Carcass Quality, Lipid Metabolism and Antioxidant Status in Broiler, Chinese Academy of Agricultural Sciences, Beijing, 2001.

15 P. Bikker, A. Dirkzwager, J. Fledderus, P. Trevisi, I. le Huërou-Luron, J. P. Lallès and A. Awati, The effect of dietary protein and fermentable carbohydrates levels on growth performance and intestinal characteristics in newly weaned piglets, J. Anim. Sci., 2006, 84, 3337-3345.

16 S. Adams, D. Che, J. Hailong, B. Zhao, H. Rui, K. Danquah and G. Qin, Effects of Pulverized Oyster Mushroom (Pleurotus ostreatus) on Diarrhea Incidence, Growth Performance, Immunity, and Microbial Composition in Piglets, J. Sci. Food Agric., 2019a, 99(7), 3616-3627.

17 S. Adams, H. Yang, D. Che, J. Hailong and G. Qin, Effects of high dietary copper supplementation on the copper accumulation and total copper content in fattening pigs, Appl. Ecol. Environ. Res., 2019b, 7, 1401-1410, DOI: 10.15666/aeer/1701_14011410.

18 C. Wenk, The role of dietary fibre in the digestive physiology of the pig, Anim. Feed Sci. Technol., 2001, 90, 21-33.

19 A. Owusu-Asiedu, J. F. Patience, B. Laarveld, A. G. Van Kessel, P. H. Simmins and R. T. Zijlstra, Effects of guar gum and cellulose on digesta passage rate, ileal microbial populations, energy and protein digestibility, and performance of grower pigs, J. Anim. Sci., 2006, 84, 843-852.

20 A. K. Agyekum and C. M. Nyachoti, Nutritional and Metabolic Consequences of Feeding High-Fiber Diets to Swine: A Review, Engineering, 2017, 3, 716-725.

21 S. Adams, C. T. Sello, G. X. Qin, D. Che and R. Han, Does dietary fiber affect the levels of nutritional components after feed formulation?, Fibers, 2018b, 6, 29, DOI: 10.3390/ fib6020029.

22 B. Ingvar, Fibre effects on intestinal functions (diarrhea, constipation and irritable bowel syndrome), Clin. Nutr. Suppl., 2004, 1, 33-38.

23 S. M. Paul, J. J. Katke and K. C. Krumhar, Bacteria-and fiber containing composition for human gastrointestinal health, 2001.

24 Y. Zhong, D. Cai, W. Cai, S. Geng, L. Chen and T. Han, Protective effect of galactooligosaccharide-supplemented enteral nutrition on intestinal barrier function in rats with severe acute pancreatitis, Clin. Nutr., 2009, 28, 575-580.

25 A. M. Brownawell, W. Caers, G. R. Gibson, C. W. C. Kendall, K. D. Lewis, Y. Ringel and J. L. Slavin, Prebiotics and the health benefits of fiber: Current regulatory status, future research, and goals, J. Nutr., 2012, 142, 962-974.

26 L. Chen, Y. Xu, X. Chen, C. Fang, L. Zhao and F. Chen, The maturing development of gut microbiota in commercial piglets during the weaning transition, Front. Microbiol., 2017, 8, 1688.

27 B. J. Kerr and G. C. Shurson, Strategies to improve fiber utilization in swine, J. Anim. Sci. Biotechnol., 2013, 4, 11.

28 C. M. Grieshop, D. E. Reece and G. C. Fahey, Nonstarch polysaccharides and oligosaccharides in swine nutrition, in Swine Nutrition, ed. A. J. Lewis and L. L. Southern, CRC Press, Boca Raton, FL, 2nd edn, 2001, pp. 107-130.
29 M. Anguita, N. Canibe, J. F. Pérez and B. B. Jensen, Influence of the amount of dietary fiber on the available energy from hindgut fermentation in growing pigs: Use of cannulated pigs and in vitro fermentation, J. Anim. Sci., 2006, 84, 27662778.

30 I. J. Wellock, P. D. Fortomaris, J. G. M. Houdijk, J. Wiseman and I. Kyriazakis, The consequences of non-starch polysaccharide solubility and inclusion level on the health and performance of weaned pigs challenged with enterotoxigenic Escherichia coli, Br. J. Nutr., 2008, 99, 520530.

31 D. Che, S. Adams, C. Wei, Q. Gui-Xin, E. M. Atiba and J. Hailong, Effects of Astragalus membranaceus fiber on growth performance, nutrient digestibility, microbial composition, VFA production, gut $\mathrm{pH}$, and immunity of weaned pigs, MicrobiologyOpen, 2018, e00712.

32 L. L. Li, F. G. Yin, B. Zhang, H. Z. Peng, F. N. Li, N. S. Zhu, D. X. Hou, Y. L. Yin, J. J. Luo, Z. R. Tang and G. Liu, Dietary supplementation with Atractylodes Macrocephala Koidz polysaccharides ameliorate metabolic status and improve immune function in early-weaned pigs, Livest. Sci., 2011, 142, 33-41.

33 C. Cheng, H. Wei, C. Xu, X. Xie, S. Jiang and J. Peng, Maternal Soluble Fiber Diet during Pregnancy Changes the Intestinal Microbiota, Improves Growth Performance, and Reduces Intestinal Permeability in Piglets, Appl. Environ. Microbiol., 2018, 84, e01047-18.

34 NRC, Nutrient Requirements of Swine, National Academies Press, Washington, DC, 11th edn, 2012.

35 K. Maenner, W. Vahjen and O. Simon, Studies on the effects of essential oil-based feed additives on performance, ileal nutrient digestibility, and selected bacterial groups in the gastrointestinal tract of piglets, J. Anim. Sci., 2011, 89, 2106-2112.

36 B. Liu, W. Wang, X. Zhu, X. Sun, J. Xiao, D. Li, Y. Cui, C. Wang and Y. Shi, Response of Gut Microbiota to Dietary Fiber and Metabolic Interaction with SCFAs in Piglets, Front. Microbiol., 2018a, 9, 2344.

37 M. J. Alfa, D. Strang, P. S. Tappia, M. Graham, G. Van Domselaar, J. D. Forbes, V. Laminman, N. Olson, P. DeGagne, D. Bray and B. L. Murray, A randomized trial to determine the impact of a digestion resistant starch composition on the gut microbiome in older and mid-age adults, Clin. Nutr., 2018, 37, 797-807.

38 J. Zhang, K. Kobert, T. Flouri and A. Stamatakis, PEAR: a fast and accurate Illumina Paired-End reAd mergeR, Bioinformatics, 2014, 30, 614-620.

39 S. Vasileiadis, E. Puglisi, M. Arena, F. Cappa, P. S. Cocconcelli and M. Trevisan, Soil bacterial diversity screening using single $16 \mathrm{~S}$ rRNA gene $\mathrm{V}$ regions coupled with multi-million read generating sequencing technologies, PLoS One, 2012, 7, e42671.

40 J. G. Caporaso, J. Kuczynski, J. Stombaugh, K. Bittinger, F. D. Bushman, E. K. Costello, N. Fierer, A. G. Pena, J. K. Goodrich, J. I. Gordon and G. A. Huttley, QIIME allows analysis of high-throughput community sequencing data, Nat. Methods, 2010, 7, 335-336. 
41 J. Ahn, R. Sinha, Z. Pei, C. Dominianni, J. Wu, J. Shi, J. J. Goedert, R. B. Hayes and L. Yang, Human gut microbiome and risk for colorectal cancer, J. Natl. Cancer Inst., 2013, 105, 1907-1911.

42 C. J. Adler, K. Dobney, L. S. Weyrich, J. Kaidonis, A. W. Walker, W. Haak, C. J. Bradshaw, G. Townsend, A. Sołtysiak, K. W. Alt and J. Parkhill, Sequencing ancient calcified dental plaque shows changes in oral microbiota with dietary shifts of the Neolithic and Industrial revolutions, Nat. Genet., 2013, 45, 450.

43 G. G. Han, J. Y. Lee, G. D. Jin, J. Park, Y. H. Choi, B. J. Chae, E. B. Kim and Y. J. Choi, Evaluating the association between body weight and the intestinal microbiota of weaned piglets via 16S rRNA sequencing, Appl. Microbiol. Biotechnol., 2017, 101, 5903-5911.

44 D. H. Parks, G. W. Tyson, P. Hugenholtz and R. G. Beiko, STAMP: statistical analysis of taxonomic and functional profiles, Bioinformatics, 2014, 30, 3123-3124.

45 N. Segata, J. Izard, L. Waldron, D. Gevers, L. Miropolsky, W. S. Garrett and C. Huttenhower, Metagenomic biomarker discovery and explanation, Genome Biol., 2011, 12, R60.

46 S. Adams, D. Che, J. Hailong, R. Han, G. Qin and K. Danquah, Dietary supplementation of pulverized Astragalus membranaceus improved performance, immunity and diarrhoea incidence in weaned piglets, Indian J. Anim. Res., 2018c, B-936.

47 A. R. Kick, M. B. Tompkins, W. L. Flowers, C. S. Whisnant and G. W. Almond, Effects of stress associated with weaning on the adaptive immune system in pigs, J. Anim. Sci., 2012, 90, 649-656.

48 R. Gresse, F. Chaucheyras-Durand, M. A. Fleury, T. Van De Wiele, E. Forano and S. Blanquet-Diot, Gut microbiota dysbiosis in post weaning piglets: understanding the keys to health, Trends Microbiol., 2017, 25, 851-873.

49 M. A. Conlon and A. R. Bird, The impact of diet and lifestyle on gut microbiota and human health, Nutrients, 2014, 7, 1744.

50 P. M. Munyaka, N. K. Nandha, E. Kiarie, C. M. Nyachoti and E. Khafipour, 517 Impact of combined beta-glucanase and xylanase enzymes on growth performance, nutrients utilization and gut microbiota in broiler chickens fed corn or wheat-based diets, Poult. Sci., 2016, 95, 528-540.

51 H. F. Wang, W. R. Yang, H. W. Yang, Y. Wang, Z. B. Yang, S. Z. Jiang and G. G. Zhang, Effects of Astragalus membranaceus on growth performance, carcass characteristics, and antioxidant status of broiler chickens, Acta Agric. Scand., Sect. A, 2010, 60, 151-158.

52 R. Gerritsen, P. van Der Aar and F. Molist, Insoluble nonstarch polysaccharides in diets for weaned piglets, $J$. Anim. Sci., 2012, 90, 318-320.

53 C. Yu, S. Zhang, Q. Yang, Q. Peng, J. Zhu, X. Zeng and S. Qiao, Effect of high fibre diets formulated with different fibrous ingredients on performance, nutrient digestibility and faecal microbiota of weaned piglets, Arch. Anim. Nutr., 2016b, 70, 263-277.
54 N. W. Jaworski, A. Owusu-Asiedu, M. C. Walsh, J. C. Mccann, J. J. Loor and H. H. Stein, Effects of a 3 strain Bacillus-based direct-fed microbial and dietary fiber concentration on growth performance and expression of genes related to absorption and metabolism of volatile fatty acids in weanling pigs, J. Anim. Sci., 2017, 95, 308-319.

55 S. L. Yuan, X. S. Piao, D. F. Li, S. W. Kim, H. S. Lee and P. F. Guo, Effects of dietary Astragalus polysaccharide on growth performance and immune function in weaned pigs, J. Anim. Sci., 2006, 82, 501-507.

56 P. Liu, J. Zhao, W. Wang, P. Guo, W. Lu, C. Wang, L. Liu, L. J. Johnston, Y. Zhao, X. Wu, C. Xu, J. Zhang and X. Ma, Dietary Corn Bran Altered the Diversity of Microbial Communities and Cytokine Production in Weaned Pigs, Front. Microbiol., 2018b, 9, 2090.

57 J. Zhao, P. Liu, Y. Wu, P. Guo, L. Liu, N. Ma, C. Levesque, Y. Chen, J. Zhao, J. Zhang and X. Ma, Dietary fiber increases the butyrate-producing bacteria and improves growth performance of weaned piglets, J. Agric. Food Chem., 2018, 66, 7995-8004.

$58 \mathrm{~K}$. W. Lee, H. Everts and A. C. Beynen, Essential oils in broiler nutrition, Int. J. Poult. Sci., 2004, 3, 738-752.

59 W. E. Swords, C. C. Wu, F. R. Champlin and R. K. Buddington, Postnatal changes in selected bacterial groups of the pig colonic microflora, Neonatology, 1993, 63, 191-200.

60 T. Gidenne, N. Jehl, M. Segura and B. Michalet-Doreau, Microbial activity in the caecum of the rabbit around weaning: impact of a dietary fibre deficiency and of intake level, Anim. Feed Sci. Technol., 2002, 99, 107-118.

61 J. Zhang, X. Chen, P. Liu, J. Zhao, J. Sun, W. Guan, L. J. Johnston, C. L. Levesque, P. Fan, T. He and G. Zhang, Dietary Clostridium butyricum Induces a Phased Shift in Fecal Microbiota Structure and Increases the Acetic AcidProducing Bacteria in a Weaned Piglet Model, J. Agric. Food Chem., 2018, 66, 5157-5166.

62 J. P. B. Freire, A. J. G. Guerreiro, L. F. Cunha and A. Aumaitre, Effect of dietary fibre source on total tract digestibility, caecum volatile fatty acids and digestive transit time in the weaned piglet, Anim. Feed Sci. Technol., 2000, 87, 71-83.

63 E. S. Chambers, A. Viardot, A. Psichas, D. J. Morrison, K. G. Murphy, S. E. Zac-Varghese, K. MacDougall, T. Preston, C. Tedford, G. S. Finlayson and J. E. Blundell, Effects of targeted delivery of propionate to the human colon on appetite regulation, body weight maintenance and adiposity in overweight adults, Gut, 2015, 64, 1744-1754.

64 D. Klein-Jöbstl, I. M. Wersen and M. Drillich, Farm characteristics and calf management practices on dairy farms with and without diarrhea: A case-control Study to investigate risk factors for calf diarrhea, J. Dairy Sci., 2014, 97, 5110-5119.

65 C. Ran, J. Hu, W. Liu, Z. Liu, S. He, B. C. T. Dan, N. N. Diem, E. L. Ooi and Z. Zhou, Thymol and carvacrol affect hybrid tilapia through the combination of direct stimulation and an intestinal microbiota-mediated effect: insights from a germ-free zebrafish model-3, J. Nutr., 2016, 146, 1132-1140. 
66 R. Lamendella, J. W. Santo Domingo, S. Ghosh, J. Martinson and D. B. Oerther, Comparative fecal metagenomics unveils unique functional capacity of the swine gut, BMC Microbiol., 2011, 11, 103.

67 D. Schokker, J. Zhang, L. L. Zhang, S. A. Vastenhouw, H. G. Heilig, H. Smidt, J. M. Rebel and M. A. Smits, Earlylife environmental variation affects intestinal microbiota and immune development in new-born piglets, PLoS One, 2014, 9, e100040.

68 S. N. Heinritz, E. Weiss, M. Eklund, T. Aumiller, S. Louis, A. Rings, S. Messner, A. Camarinha-Silva, J. Seifert, S. C. Bischoff and R. Mosenthin, Intestinal microbiota and microbial metabolites are changed in a pig model fed a high-fat/low-fiber or a low-fat/high-fiber diet, PLoS One, 2016, 11, e0154329.

69 J. Hu, Y. Nie, J. Chen, Y. Zhang, Z. Wang, Q. Fan and X. Yan, Gradual changes of gut microbiota in weaned miniature piglets, Front. Microbiol., 2016, 7, 1727.

70 G. G. Mateos, E. Jiménez-Moreno, M. P. Serrano and R. P. Lázaro, Poultry response to high levels of dietary fiber sources varying in physical and chemical characteristics, $J$. Appl. Poult. Res., 2012, 21, 156-174.
71 M. Kim, J. Kim, L. A. Kuehn, J. L. Bono, E. D. Berry, N. Kalchayanand, H. C. Freetly, A. K. Benson and J. E. Wells, Investigation of bacterial diversity in the feces of cattle fed different diets, J. Anim. Sci., 2014, 92, 683-694.

72 A. El Kaoutari, F. Armougom, J. I. Gordon, D. Raoult and B. Henrissat, The abundance and variety of carbohydrateactive enzymes in the human gut microbiota, Nat. Rev. Microbiol., 2013, 11, 497.

73 S. A. Frese, K. Parker, C. C. Calvert and D. A. Mills, Diet shapes the gut microbiome of pigs during nursing and weaning, Microbiome, 2015, 3, 28.

74 R. E. Ley, P. J. Turnbaugh, S. Klein and J. I. Gordon, Microbial ecology: human gut microbes associated with obesity, Nature, 2006, 444, 1022-1023.

75 P. J. Turnbaugh, R. E. Ley, M. A. Mahowald, V. Magrini, E. R. Mardis and J. I. Gordon, An obesity-associated gut microbiome with increased capacity for energy harvest, Nature, 2006, 444, 1027-1031.

76 A. W. Walker, J. Ince, S. H. Duncan, L. M. Webster, G. Holtrop, X. Ze, D. Brown, M. D. Stares, P. Scott, A. Bergerat and P. Louis, Dominant and diet-responsive groups of bacteria within the human colonic microbiota, ISME J., 2011, 5, 220. 